\title{
LAS LEGUMINOSAS CESALPINIOIDEAS DE LA PROVINCIA DE SANTA FE
}

\author{
LUCHETTI, A. M. ${ }^{1}$ \& EXNER, E. de L. ${ }^{1}$
}

\begin{abstract}
RESUMEN
Como contribución al estudio de la flora de Santa Fe, se presenta la revisión sinóptica de las Leguminosas Cesalpinioideas que crecen espontáneamente en el territorio de esta provincia. En total se incluyen 19 especies distribuidas en 9 géneros. Los siguientes taxones: Senna aphylla (Cav.) H.S. Irwin \& Barneby y Senna spectabilis (DC.) H. S. Irwin \& Barneby, son excluidos de esta flora. Para cada género se proporcionan claves que permiten el reconocimiento de sus especies. Para cada taxón se incluye su nombre correcto aceptado por la comunidad botánica y, cuando corresponde, su basónimo; nombres vernáculos, ejemplares de referencia, hábito, iconografía, distribución geográfica y observaciones fenológicas y ecológicas.
\end{abstract}

Palabras clave: Leguminosae, Cesalpinioideae, Santa Fe.

\section{ABSTRACT}

\section{The Leguminosas Cesalpinioideas of the Province of Santa Fe.}

A review of the Leguminosae Cesalpinioideae growing naturally in the territory of Santa Fe province, Argentina, is presented as a contribution to the study of the flora of this province. A total of 19 species belonging to 9 genera are included. The following taxa are excluded from the flora of Santa Fe: Senna aphylla (Cav.) H.S. Irwin \& Barneby y Senna spectabilis (DC.) H. S. Irwin \& Barneby. For each genus, keys for the recognition of its species are provided. For each taxon, the following items are included: the correct name acceted by the botanical community and, when applicable, its basionym; vernacular names, a reference specimen, habitat, iconography, geographical distribution and ecological and phenological observations.

Key words: Cesalpinioideae, Leguminosae, Santa Fe.

1.- Cátedra de Botánica Sistemática Agronómica. Facultad de Ciencias Agrarias. Universidad Nacional del Litoral. Kreder 2805. (3080) Esperanza, provincia de Santa Fe. Argentina. Email: amluche@arnet.com.ar Manuscrito recibido el 12 de abril de 2018 y aceptado para su publicación el $1^{\circ}$ de agosto de 2018.

Luchetti, A. M.. \& Exner, E. de L. Las leguminosas Cesalpinioideae en la provincia de Santa Fe. FAVE Ciencias Agrarias 17 (2): 7-21. CC BY-NC-SA 4.0 (c) (i) (2) 


\section{INTRODUCCIÓN}

Leguminosae o Fabaceae constituye una importante familia de distribución cosmopolita, la tercera más amplia del reino vegetal, después de Asteraceae y Orchidaceae (Christenhusz, M.J.M. \& J.W. Byng., 2016). Desde el punto de vista económico, su relevancia sólo es superada por Poaceae. Sus 770 géneros y más de 19.500 especies (LPWG, 2017), se agrupaban en 3 subfamilias tradicionalmente conocidas como: Cesalpinioideae DC., Mimosoideae DC. y Papilionoideae DC., clasificación fundada en caracteres morfológicos, esencialmente florales. Sin embargo, las nuevas hipótesis filogenéticas basadas en marcadores plastidiales, sustentan la existencia de 6 subfamilias: Cercidioideae LPWG, Detarioideae Burmeist., Dialioideae LPWG, Duparquetioideae LPWG, Cesalpinioideae DC. y Papilionoideae DC. (LPWG, 2017).La subfamilia Cesalpinioideae sensu lato es pantropical, y sus especies están presentes en un amplio rango de ambientes: selvas, bosques, sabanas y semidesiertos, especialmente de África, Asia y América; pocas habitan en zonas templadas (Ulibarri, 2008).En Argentina, esta subfamilia está representada por 15 géneros (Zuloaga et al. 2008) y 73 especies (www.floraargentina.edu.ar X-2018). Para la provincia de Santa Fe, Ragonese (1941) cita 6 géneros y 9 especies; posteriormente, Pensiero et al. (2005) mencionan 9 géneros y 21 especies. En la presente contribución se citan para Santa Fe, 9 géneros y 19 especies de la subfamilia Cesalpinioideae; los restantes taxones que actualmente forman parte de esta subfamilia (LPWG, 2017), no se incluyen, ya que fueron tratados con anterioridad por Luchetti (2014) en la revisión de la subfamilia Mimosoideae. Se suministran claves que permiten, para cada género, el reconocimiento de sus espe- cies. Además, se aporta información acerca del hábito, ecología, fenología y distribución geográfica de cada entidad considerada.

\section{MATERIAL Y MÉTODOS}

Para el abordaje de esta revisión se siguió la metodología clásica vinculada a estudios taxonómicos; se realizaron observaciones de material vivo y, especialmente, de especímenes de herbario depositados en las siguientes instituciones: Universidad Nacional del Litoral: Herbario “Arturo E. Ragonese” (SF); Universidad Nacional de Rosario: Herbario "J. P. Lewis" (UNR) y del Instituto de Botánica Darwinion (SI). Por razones de espacio, no se detalla la sinonimia, incluyéndose -cuando corresponde- los basónimos y, en cuanto al material estudiado sólo se cita un ejemplar de referencia para cada departamento. En lo que respecta a la iconografía, se citan cronológicamente las obras que incluyen ilustraciones y/o fotografías de los taxones tratados. Los herbarios se nombran conforme a las siglas del Index Herbariorum, mientras que para las autoridades correspondientes a cada taxón, se siguió a Brummit \& Powell (1992). Los títulos de las revistas citadas han sido abreviados de acuerdo a Bridson (2004). Las claves para el reconocimiento de cada género pueden ser consultadas en Pensiero et al. (op.cit). Tanto los géneros como las especies, se presentan ordenados alfabéticamente. En relación a la distribución geográfica de las especies, sólo se indica la correspondiente a la provincia de Santa Fe. El tratamiento de cada género se basa en las siguientes revisiones taxonómicas: Bauhinia Plum, ex L.: Fortunato (1986 y 1997); Ulibarri (1997); Chamaecrista Moench: Burkart (1952 y 1987), Irwin \& Barneby. (1982); Erytrosthemon Klotzsch: 
Burkart (1936 y 1952), Ulibarri (1996 y 1997), Gagnon et al. (2016); Gleditsia: Burkart (1987), Ulibarri (1997); Hoffmanseggia Cav.: Ulibarri (1996 y 1997); Libidibia (DC.) Schltdl.: Burkart (1936 y 1952), Ulibarri (1996 y 1997), Gagnon et al. (2016); Parkinsonia L.: Burkart \& Carter. (1976), Ulibarri (1997), Hawkins et al. (1999); Peltophorum (Vogel) Benth.: Burkart (1952 y 1987), Ulibarri (1997); Senna Mill.: Burkart (1946, 1952 y 1987), Irwin \& Barneby. (1982), Robbiati et al. (2014).En lo que respecta a la delimitación de las provincias fitogeográficas, se sigue a Cabrera (1994) y a Pensiero et al. (2005).

\section{RESULTADOS}

1. Bauhinia Plum, ex L.

1. Bauhinia forficata subsp. pruinosa (Vogel) Fortunato \& Wunderling, Darwiniana 27: 550. 1986.

Basónimo: Bauhinia pruinosa Vogel, Linnaea 13: 301. 1839.

Nombres vernáculos: "Pata de vaca", "Pezuña de vaca”, "Pata de buey", "Falsa caoba”, "Pata de chivo", "Caoba del país”, "Palo buey”, "Mahogani”.

Iconografía: Burkart A. (1952: 159, Fig. 26; 1987: VI. 3: 510, Fig. 229; 1987: 510, Fig. 229); Jozami \& Muñoz: 1984: Pag. 137138); Fortunato R. (1986: 27 (1-4) 551, Fig. 6); Parodi (1987: 489, Fig. 128); Muñoz et al. (1993: Pag. 61 y 63); Dimitri et al. (1998: Pág. 86-87); Juárez de Varela F. (2001: 3, Lám. 1); Hurrell \&Lahitte (2002: 81).

Distribución geográfica: Dptos. General Obligado, La Capital y Rosario.

Ejemplares de referencia: Covas \& Ragonese 4680 (SF), Ragonese 2199 (SF), Franceschi 1125 (UNR).

Hábito y ecología: Árbol o arbusto de 6 a
$8 \mathrm{~m}$ de altura, con tallos ramificados, ramas con aguijones cónicos, geminados y raíces gemíferas. En la provincia se lo ha coleccionado y observado en zonas urbanas y periurbanas. Cultivada como ornamental y para cercos vivos, suele asilvestrarse y formar densos arbustales en terrenos baldíos y ambientes modificados. Florece en el verano y parte del otoño, fructifica desde febrero a marzo; sus frutos perduran hasta julio en la planta.

Observación: Valorada como ornamental por sus grandes flores blancas, medicinal y, escasamente, como maderable. Si bien se trata de una especie nativa de Argentina, en la provincia solo se la ha colectado en ambientes donde fue cultivada o escapada de cultivo.

\section{Chamaecrista Moench Clave para la diferenciación de las especies}

1 Hierbas postradas. Hojas uniyugas, casi glabras, con folíolos obovales de 1-2 (-2,5) cm long. Pecíolos sin glándulas nectaríferas. Pedúnculos filiformes, más largos que las hojas, generalmente unifloros. Flores menores de $1 \mathrm{~cm}$ long. Androceo 5, a veces con 1-3 estaminodios 3. Ch. rotundifolia.

1' Hierbas erguidas desde la base o ascendentes. Hojas 4-25 yugas, pubescentes. Pecíolos con glándulas nectaríferas pateliformes, sésiles o estipitadas. Pedúnculos más cortos que las hojas, 1-3 (-4) floros. Flores de (0,4-) 0,6 - $2 \mathrm{~cm}$ long. Androceo 10 (-9), sin estaminodios

2 Hojas 4-15 yugas, levemente pubescentes, de hasta $5 \mathrm{~cm}$ long. Pecíolos con glándulas nectaríferas sésiles. Folíolos de hasta $1 \mathrm{~cm}$ long. con nervadura principal subcentral. Flores de 1,5-2 cm long. .. .. Ch. repens. 
A. M. Luchetti et al.

2' Hojas 10-25 yugas netamente pubescentes, de hasta $8 \mathrm{~cm}$ long. Pecíolos con glándulas nectaríferas sésiles o levemente estipitadas (estípite de hasta 0,5 mm long.). Folíolos de hasta 1,5 cm long., con nervadura principal submarginal. Flores de hasta 0,6 cm long. nictitans var. patellaria.

1. Chamaecrista nictitans var. patellaria (DC. ex Collad.) Kartesz \& Gandhi, Mem. New York Bot. Gard., 35: 814. 1982. Basónimo: Cassia patellaria DC. ex Collad., Hist. Nat. Méd. Casses 125, pl.16.1816. Iconografía: Burkart (1952: 163, Fig. 29, sub. nom. Cassia patellaria); Arbo \& Tressens

(2002: 369, Fig. 6.283); Fabbroni (2010: 40, Lám. 14).

Distribución geográfica: Dptos. General Obligado, 9 de Julio y San Javier.

Ejemplar de referencia: Pensiero 8919 (SF), Pire 958 (UNR), Pensiero 11799 (SF).Hábito y ecología: Hierba anual, erguida, ramificada desde la base, de 0,2 a 0,8 $\mathrm{m}$ de altura. Crece en ambientes de la Cuña Boscosa, de la provincia Chaqueña y en la zona norte de la provincia del Espinal, integrando el estrato herbáceo de los bosques, y en bordes de caminos. Florece en primavera, fructifica en el verano.

2. Chamaecrista repens (Vogel) H. S. Irwin \& Barneby, Mem. New York Bot.Gard., 35: 742. 1982.

Basónimo: Cassia repens Vogel, Gen.Cass. Syn. 60.1837.

Iconografía: Burkart (1987: 523, Fig. 236); Arbo \& Tressens (2002: 369, Fig. 6.282); Ulibarri (2008: 113, Fig. 6); Fabbroni (2010: 35, Lám. 13). Distribución geográfica: Dptos. 9 de Julio, San Javier y Vera.
Ejemplar de referencia: Pensiero 3002 (SF), Ragonese 2700 (SF), Pire \& Nisensohn 318 (UNR).

Hábito y ecología: Hierba perenne, rizomatosa, ascendente-erecta de hasta $40 \mathrm{~cm}$ de altura, muy ramificada desde la base del tallo, con rizoma delgado. Habita en el centro-oeste de la provincia Chaqueña y en sitios húmedos, próximos a cursos de agua y a la vera de caminos, del centro delaprovincia Paranense. Florece y fructifica en primavera y verano.

3. Chamaecrista rotundifolia (Pers.) Greene var. rotundifolia, Pittonia 4(20D): 31. 1899.

Basónimo: Cassia rotundifolia Pers., Syn. Pl. 1: 456. 1805.

Iconografía: Irwin \& Barneby (1982: 732, Fig. 47); Burkart (1987: 525, Fig. 237).

Distribución geográfica: Dptos Garay, General Obligado, 9 de Julio y San Javier. Ejemplar de referencia: Pensiero 6437 (SF), Franceschi 885 (UNR), Ragonese 2964 (SF), Ragonese 2700 (SF).

Hábito y ecología: Hierba perenne, con tallos rastreros no radicantes, o ascendentes y raíz pivotante profunda. Se la encuentra en suelos arenosos de la provincia Chaqueña y del Espinal, transicionales con la provincia Paranense. Se la ha coleccionado en zonas bajas, integrando comunidades de Polygonum L. (cataizales) y de Ludwigia L. (verdolagales) y el estrato herbáceo de bosques de Vachellia caven (Molina) Seigler \& Ebinger (espinillares).

\section{Erythrostemon Klotzsch}

1. Erythrostemon gilliesii (Hook.) Klotzsch, Ic. Pl. Rar. Horti. Berol. 2 (3): 97, t. 39. 1844.

Basónimo: Poinciana gilliesii Wall. ex Hook., Bot. Misc. 1: 129-131, pl. 34. 1830. 
Nombres vernáculos: "Barba de chivo", subfalcadas, leñosas, negruzcas a la madu“Lagaña de perro”, "Barba de viejo”, rez, de 4-10 x 2,5-5 cm .......................1. "Mal de ojo", "Algarrobilla”, "Discipli- G. amorphoides var. amorphoides na de monja”, “Espiga de amor”, "Flor 1' Folíolos elíptico-ovados a lanceolade seda", "Picha de perro", "Piscala”. dos, levemente crenados. Espinas comIconografía: Bianco \& Cantero (1992: 132, primidas, generalmente 1-2 ramificadas, sub. nom. Caesalpinia gilliesi); Burkart con aspecto de cruz. Flores de 4-6 mm. (1936: 80, f. 1 i, 91, f. 3 b-b’ Lám. 5 AC; Legumbres largas, subrectas o espirala1987: 527, Fig. 238; 1987: 527, Fig. 238, das, coriáceas, castaño-rojizas, de 15-42 x sub. nom. Caesalpinia gilliesi); Jozami \& Muñoz (1984: 145, sub. nom. Caesalpinia gilliesi ); Muñoz, Ross \& Gracco (1993: 75, sub. nom. Caesalpinia gilliesi); Ulibarri (1994: 280, Fig. 316; 1996: 321, Fig. 4, sub. nom. Caesalpinia gilliesi ); Hurrell \& Lahitte (2002: 59, sub. nom. Caesalpinia gilliesi ); Gagnon et al.(2016: 116, Fig. 41). Distribución geográfica: Dptos. 9 de Julio, Rosario y San Cristóbal.

Ejemplar de referencia: Vegetti 955 (SF), Lewis \& Collantes 830 (UNR), Ragonese 3040 (SF).

Hábito y ecología: Arbusto inerme, de 0,80 a 3 m de altura, con ramas de pubescencia glandulosa, rojiza. Se lo ha coleccionado en suelos secos del oeste de las provincias fitogeográficas Chaqueña y del Espinal. Además, se lo cultiva en jardines y plazas, por lo que, escapado de cultivo, se lo puede observar como subespontáneo en otras áreas de la provincia. Florece desde octubre a marzo. Fructifica en el verano. Observación: Recomendada en xerojardinería (Eynard et al., 2017), ornamental, medicinal.

\section{Gleditsia L. \\ Clave para diferenciar las especies}

1 Folíolos ovados-subelípticos a subobovados, netamente crenados. Espinas redondeadas o de base comprimida, 1-3 ramificadas. Flores de 6-7 mm. Legumbres breves,

2. G. triacanthos.

1. Gleditsia amorphoides (Griseb.) Taub. var. amorphoides, Ber. Deutsch. Bot. Ges.10: 638. 1892.

Basónimo: Garugandra amorphoides Griseb., Symb. Fl. Argent.: 96. 1879.

Nombres vernáculos: "Espina corona”, "Espina de corona”, "Coronillo blanco"; “Ivopé”; “Carandí”; “Cambá nambí”.

Iconografía: Burkart (1952: 186, Fig. 38; 1987: 534, Fig. 241); Dimitri et al. (1997 b: 30-31); Pensiero \& de la Peña (19992000: 181); Hurrell \& Lahitte (2002: 71); Peña-Chocarro et al. (2006: 204).

Distribución geográfica: Dptos. General Obligado y Vera.

Ejemplar de referencia: Pensiero 8141 (SF), Pensiero 8677 (SF).

Hábito y ecología: Árbol espinoso, caducifolio, de 3 a 15 (-20) m de altura, con espinas de distribución irregular, a veces escasas, en ramas jóvenes, y agrupadas formando ramilletes densos en el tronco de hasta $25 \mathrm{~cm}$ de longitud. Frecuente en los bosques de las provincias Chaqueña y Paranense. Florece a principios del verano, coleccionado con frutos desde enero a junio.

Observaciones: Se utiliza su madera pesada, dura y veteada -aunque poco duradera al aire libre - en construcciones, carpintería y ebanistería, y como combustible. Sus frutos son forrajeros y contienen taninos y saponinas, sirven para curtir, lavar y como in- 
sectífugos; el mucílago de las semillas tiene valor industrial: se extrae y se usa en la fabricación de dulces de batata y guayaba.

2. Gleditsia triacanthos L., Sp. Pl 2: 1056-1057. 1753.

Nombres vernáculos: “Acacia negra”, “Acacia de 3 espinas”, "Espina de Cristo”, "Espina corona de Cristo”, "Algarroba turca”. Iconografía: Burkart: (1952: 186, Fig. 38; 1967: 463: 1987: 536, Fig. 242); Parodi (1987: 493, Fig. 199); Hurrell \& Lahitte (2002: 67, 69).

Distribución geográfica: Dptos. Iriondo, La Capital,LasColonias, RosarioySanLorenzo. Ejemplar de referencia: Ragonese 4068 (SF), Exner 889(SF).

Hábito y ecología: Árbol caducifolio, de 18 $\mathrm{m}$ de alto, ramas rojizas con espinas gruesas, con 1-2 hasta muchas ramificaciones (hay variedades sin espinas). Vive en suelos sueltos, húmedos y arcillosos; resiste anegamientos temporarios y estacionales. En Santa Fe se comporta como una agresiva especie invasora de banquinas, caminos rurales y áreas naturales del Espinal, particularmente en el departamento Las Colonias. También se la ha observado como subespontánea en sitios de la región Pampeana. Observaciones: Especie introducida, procedente de Estados Unidos de América. Naturalizada en el Centro y Este de nuestro país, incluyendo la provincia de Santa Fe. Ornamental y forestal, rústica, de buena madera para postes y ruedas; melífera y forrajera (el ganado vacuno consume sus frutos y dispersa las semillas, que se escarifican en el tracto digestivo). Los frutos flotan en el agua, por lo que al desbordar los ríos avanza su área de dispersión. Las semillas poseen un mucílago de valor industrial, aunque de menor calidad que el de G. amorphoides. Debido a su carácter invasor se desaconseja su utilización en nuestra provincia.

\section{Hoffmanseggia Cav.}

1. Hoffmanseggia glauca (Ortega) Eifert, Sida 5(1): 43. 1972.

Basónimo: Larrea glauca Ortega, Nov. Pl. Descr. Dec. 2: 15-16. 1797.

Nombres vernáculos: "Porotillo", "Porotillo silvestre”, “Algarrobilla fina”, "Camincha”, "Papa cuchi”.

Iconografía: Burkart (1952: 177, Fig.53 sub. nom. H. falcata); Ruiz Leal (1972: 207, Lám. 64); Ulibarri (1979: 141-142, Fig. 1-4, 1984: 222, Fig. 573, 1996: 336, Fig. 6). Distribución geográfica: Dptos.: San Cristóbal y Vera.

Ejemplar de referencia: Stofella 254 (UNR), Pire \& Nisensohn 279. Hábito y ecología: Hierba caulescente o subacaule, de 5 a $30 \mathrm{~cm}$ de altura, generalmente pubescente. Crece en áreas de las provincias Chaqueña y del Espinal, en suelos secos, salobres, y en arenosos y húmedos, próximos a cursos de agua y en banquinas altas. Florece desde octubre a marzo; coleccionado con frutos en diciembre y abril.

\section{Libidibia (DC.) Schltdl.}

1. Libidibia paraguariensis (D. Parodi) G.P. Lewis, Mabberley’s Pl. -Book (ed.3) 1021. 2008.

Basónimo: Acacia paraguariensis D. Parodi, Revista Farm. 3: 7. 1862.

Nombres vernáculos: “Guayacán”, Guayacán negro”, "Ibirá-berá”, “Guayacán del Chaco”.

Iconografía: Burkart (1936: 80, Fig. 1 f-h; 95: 4b- b’; Lám. 1 A-B, sub. nom. Caesalpinia melanocarpon); Dimitri et al. (1997 a: 36-37, sub nom. Caesalpinia paraguariensis); Hurrell \& Lahitte (2002: 73, sub nom. Caesalpinia paraguariensis); Peña et al. (2006: 232 sub nom. Caesalpinia 
paraguariensis); Eynard C. et al. (2017: 261, sub nom. Caesalpinia paraguariensis); Gagnon et al.(2016: 97, Fig. 32, B). Distribución geográfica: Dptos. General Obligado, 9 de Julio, San Javier y Vera. Ejemplar de referencia: Pensiero 8897 (SF), Ragonese 3096 (SF), Pensiero 4951 (SF), Pensiero 8923 (SF).

Hábito y ecología: Árbol inerme, glabro, de 6 a $15(-20) \mathrm{m}$ de altura, ramas arqueadas, péndulas en el ápice, troncos breves, madera dura y corteza con placas grisáceo-verdosas que se desprenden y dejan ver manchas irregulares claras, lisas, grises o verdosas. Crece en la provincia Chaqueña, integrando el estrato arbóreo de la Cuña Boscosa, y los quebrachales más xerófilos del Dorso Occidental Subhúmedo (Pensiero et al., 2005). Florece de octubre a diciembre. Coleccionado con frutos desde diciembre a junio. Observación: Su madera dura y pesada es muy valorada por su resistencia a la intemperie, como combustible y fuente de taninos (al igual que sus frutos). Planta tintórea (frutos y semillas). Medicinal (corteza y hojas). Forrajera. Potencialmente como melífera.

\section{Parkinsonia L.}

\section{Clave para diferenciar las especies}

1 Hojas con pinnas de 9 a 30 (38) cm long., con más de 20 pares de foliólulos muy separados entre sí (distancia entre foliólulos mayor que la longitud de los mismos). Espinas 3, nodales, la mediana recta (raquis foliar abreviado) y las laterales recurvadas. Legumbres irregularmente torulosas, con constricciones entre las semillas, apiculadas, castaño-cobrizas

1. Parkinsonia aculeata
1'Hojas con pinnas de 0,5-1,2 (2) cm long., con raquis filiforme y 5 a 8 (11) pares de foliólulos próximos entre sí (distancia menor que la longitud de los mismos). Legumbres linear a ligeramente elípticas, aplanadas, agudas, castaño amarillentas.

2. Parkinsonia praecox

1. Parkinsonia aculeata L., Sp. Pl.1: 375. 1753.

Nombres vernáculos: "Cina -cina”, "Palo verde”, "Retamo rojo”, "Brea de agua”.

Iconografía: Burkart (1952: 182, Fig.36; 1967: 461, Fig. 145; 1987: 532, Fig.240); Ulibarri (1994: 281, Fig. 319); Pensiero \& de la Peña (1999-2000: 176); Hurrell \& Lahitte (2002: 63, 65); Peña-Chocarro et al. (2006: 244).

Distribución geográfica: Dptos.: General Obligado, La Capital, Las Colonias, 9 de Julio, Rosario, San Cristóbal, San Jerónimo, San Justo, San Martín y Vera.

Ejemplar de referencia: Pensiero 7783 (SF), Marino 97 (SF), Pensiero 1098 (SF), Pensiero 3377 (SF), Franceschi 1032 (UNR), Covas \& Ragonese 4520 (SF), Tedone 5554 (SF), Courauld \& Ragonese 2085 (SF), Vegetti 354 (SF), Pire \& Nisensohn 278 (UNR).

Hábito y ecología: Arbusto o árbol heliófilo, espinoso, de 2-6 (8) m de altura, caducifolio o semicaducifolio, de copa aparasolada, poco densa, ramas largas algo péndulas. Su presencia siempre está asociada a terrenos bajos, generalmente inundables, a lo largo de los cursos de ríos y arroyos, preferentemente en suelos arenosos y sueltos, aunque puede tolerar varios meses de sequía y casi cualquier tipo de suelo. Es típico de la provincia del Espinal, siendo la especie emblemática de los bosques de la cuenca del Río Salado; también se lo halla en las provincias Chaqueña, Paranense 
y Pampeana (valle de inundación del río Carcarañá) donde crece en zonas bajas con suelos halohidromórficos, integrando sabanas junto a Vachellia caven (Molina) Seiger \& Ebinger (aromito, espinillo), Prosopis nigra (Griseb.) Hieron. (algarrobo negro) y Geoffroea decorticans (Gillies ex Hook. \& Arn.) Burkart var. decorticans (chañar). Florece desde mediados de octubre a marzo. Fructifica de noviembre a abril.

Observaciones: Se cultiva como cerco vivo u ornamental por su bella y abundante floración amarilla, su rusticidad y su rápido crecimiento. Además es una especie melífera, medicinal y forrajera. Su madera semipesada se utiliza en carpintería y para leña. En climas cálidos se emplea para el control de la erosión.

Parkinsonia praecox (Ruiz \& Pav. ex Hook.) Hawkins, Pl. Syst. Evol. 216: 63. 1999.

Basónimo: Caesalpinia praecox Ruiz \& Pav. ex Hook., Fl. Peruv. 4 pl. 376. 1830.

Nombres vernáculos: "Brea", "Palo verde", "Chañar brea".

Iconografía: Haene-Aparicio (2001: 72, sub nom. Cercidium praecox); Bianco et al. (2001: 118, sub. nom. Cercidium praecox); Demaio et al. (2002: 84-85, sub. nom. Cercidium praecox); Peña-Chocarro et al. (2006: 234, 235, sub. nom. Cercidium praecox); Eynard et al. (2017: 265, sub. nom. Cercidium praecox).

Distribución geográfica: Dptos. 9 de Julio y San Cristóbal.

Ejemplar de referencia: Ragonese 2929 (SF), Martinez 4659 (SF).

Hábito y ecología: Árbol o arbusto de 2 a 6 (-10) $\mathrm{m}$ de altura, caducifolio, de copa aparasolada, rala y de tronco corto, muy ramificado, corteza lisa, delgada y verdosa, que por incisiones segrega una sustancia gomosa ("goma ámbar”). Integra el estrato arbóreo de los bosques del Dorso Occidental en el sector santafesino de la provincia Chaqueña y áreas de transición con el oeste del Espinal. Florece de septiembre a noviembre. Fructifica de noviembre a marzo.

Observaciones: Su madera se utiliza como combustible y escasamente en carpintería; la goma tiene aplicación industrial y alimentaria; medicinal; melífera; ornamental. Es considerada recurso forrajero de "emergencia".

\section{Peltophorum (Vogel) Benth.}

1. Peltophorum dubium (Spreng.) Taub., Nat. Pflanzenfam 3(3): 176. 1892.

Basónimo: Caesalpinia dubia Spreng., Syst. Veg. 2: 343. 1825.

Nombres vernáculos: "Ibirá pitá", "Ibirapuitá”, "Ibirá-pitá guazú”, “Cañafístula”, "Árbol de Artigas".

Iconografía: Burkart (1987: 3: 529, Fig. 239); Dimitri et al. (1997 b: 48-49); Hurrell \& Lahitte (2002: 77, 79); Peña-Chocarro et al. (2006: 246).

Distribución geográfica: Dptos: General Obligado, Garay y San Javier.

Ejemplar de referencia: Pensiero 8965 (SF).

Hábito y ecología: Árbol majestuoso, de 10$30 \mathrm{~m}$ de altura, copa amplia, follaje tardíamente caduco. Crece en bosques higrófilos del norte y centro de la provincia Paranense. Requiere suelo húmedo, arenoso, fértil, profundo, bien drenado y altas temperaturas. Florece desde mediados de diciembre a abril y fructifica desde enero a mayo.

Observación: Especie cultivada como ornamental por su follaje verde oscuro y su vistosa floración amarilla. Su madera dura y pesada, de hermoso veteado, se utiliza en 
carpintería, ebanistería y en construcciones civiles y navales.

\section{Senna Mill. Clave para diferenciar las especies}

1 Plantas con hojas (a veces prontamente caducas en $S$. chloroclada). Legumbres dehiscentes o indehiscentes, secas o pulposas. Arbustos, subarbustos o hierbas

2 Hojas con glándula nectarífera gruesa, basal, en la cara superior del pecíolo (no entre los folíolos), 4-8 yugadas. Legumbres ascendentes o erectas (raro subpéndulas), dehiscentes, sin elasticidad, secas. Subarbustos o hierbas

3 Plantas generalmente anuales. Hojas 4-6 yugadas, pecíolos de 2,5-5 cm long., folíolos glabros, oval-lanceolados, Racimos breves, axilares y el terminal más corto que las hojas acompañantes ...4. S. occidentalis

3'Subarbustos perennes. Hojas 5-8 yugadas, pecíolos generalmente de $0,5-2 \mathrm{~cm}$ long folíolos subglabros, escabroso-pilosos a pubescentes en el envés, lanceolados a oblongo-lanceolados, agudos en el ápice. Racimos mayores, corimbosos en el ápice y superando o igualando a las hojas superiores

6. S. scabriuscula

2' Hojas sin glándula nectarífera basal, pero sí con 1 o varias glándulas raqueales en el punto de inserción de los folíolos inferiores (primer par y a veces subsiguientes), 1-5 yugadas. Legumbres generalmente péndulas, cilíndricas, indehiscentes o dehiscentes, secas o subpulposa. Arbustos ... 4

4 Legumbres cilindroides o deprimido-cilíndricas, generalmente péndulas, apergaminadas o sub-pulposas, indehiscentes o tardíamente dehiscentes, con falsos tabiques transversales entre las numerosas semillas, también transversales. Hojas 2-5 yugadas, folíolos hasta de 5,5 cm long.. ..

5 Arbustos pubescentes. Hojas 3-5 yugadas, folíolos oblongo-lanceolados, de envés muy pubescente. Legumbres indehiscentes. Racimos breves (axilares y apicales), corimbosos 3. S. morongii

5' Arbustos glabros o escasamente pilosos. Hojas 2-5 yugadas, glabras. Legumbres generalmente indehiscentes. Racimos amplios, compuestos ..6

6 Hojas 3-5 yugadas. Folíolos elípticos u obovales, levemente discolores. Legumbres cilindroides, gruesas, netamente deprimidas, con suturas más o menos impresas, indehiscentes, subpulposas Semillas biseriadas. .5. S. pendula var. paludicola

6’Hojas 2-3 yugadas. Folíolos agudos o subagudos, más angostos. Legumbres cilíndricas, delgadas, sin suturas impresas, tardíamente dehiscentes o indehiscentes Semillas uniseriadas

\section{S. corymbosa}

4' Legumbres subcilíndricas, poco comprimidas, erguidas o de base ascendente, secas, dehiscentes o rara vez indehiscentes. Semillas oblicuas. Hojas 1-2 yugadas, folíolos hasta de $2 \mathrm{~cm}$ long.-...............

1. S. chloroclada

1’Plantas áfilas a la antesis. Legumbres dehiscentes, sin elasticidad, secas. Semillas oblicuas. Ramas glabras. Racimos axilares agrupados a lo largo de ramitas terminales. Arbustos o subarbustos 7. S. spiniflora 
A. M. Luchetti et al.

1. Senna chloroclada (Harms) H.S. Irwin coleópteros (Hurrell \& Lahitte, 2002).

\& Barneby, Mem. New York Bot. Gard. 35: 3. Senna morongii (Britton) H. S. Irwin 565. 1982.

\& Barneby, Mem. New York Bot. Gard. 35:

Basónimo: Cassia chloroclada Harms, 364. 1982.

Meded. Rijks-Herb. 27: 37. 1915. Basónimo: Cassia morongii Britton, Ann. Nombre vernáculo: "Sacha alfa”.

N.Y. Acad. Sci. 7: 91. 1892.

Iconografía: Bravo (1981: 263, Fig. 2). Nombres vernáculos: “Sen”, "Sen del burHábito y ecología: Arbusto o subarbusto de ro”, "Pito”, "Pitocanuto”, “Algarrobo de lo 0,5 a 1.5 m de altura, muy ramificado, con ramas de color verde, apoyantes o postradas y hojas prontamente caducas. Florece y fructifica desde agosto a abril (Bravo, 1978). Observación: Citado por Zuloaga \& Morrone (1999), Zuloaga et al (2008) y www. floraargentina.edu.ar X-2018. No fueron hallados especímenes de referencia.

2. Senna corymbosa (Lam.) H. S. Irwin \& Barneby, Mem.New York Bot. Gard. 35: 397. 1982.

Basónimo: Cassia corymbosa Lam., Encycl. 1(2): 644. 1785.

caranchos".

Iconografia: Burkart (1987: 515, Fig. 231); Ulibarri (1994: 276, Fig. 310); Barboza et al. (2006: 752, Fig. 287). Distribucion geográfica: Dptos. General Obligado, 9 de julio, San Cristóbal y Vera. Ejemplar de referencia: Pensiero 2604 (SF), Ragonese 3214 b (SF), D'Angelo 616 (SF), Pensiero 11210 (SF). Hábito y ecología: Arbusto perenne de 1-2 $\mathrm{m}$ de altura, erguido, con ramas y hojas pubescentes, tallos cilíndricos, macizos, muy ramificados. Crece en la provincia Chaqueña Nombres vernáculos: "Sen de campo”, y el oeste del Espinal; integra el estrato ar"Rama negra”, "Mata negra”, "Café de la bustivo de los bosques, habita en abras entre India”, “Cañafístula”. matas de Sporobolus R. Br. (espartillares), Iconografía: Jozami \& Muñoz: 1984 (139); Burkart (1987: 518, Fig. 233); Muñoz et al. (1993: 83); Hurrell \& Lahitte (2002: 87); Barboza et al. (2006: 750, Fig. 286), Eynard et al. (2017: 2017).

Distribución geográfica: Dptos. General Obligado y Vera.

Ejemplar de referencia: Pensiero \&Tivano 3117 (SF), Tedone 4986 (SF). Hábitoyecología: Arbusto de 0,8 a 4 m de altura, semiperennifolio, tallos generalmente apoyantes y ramas rojizas o negras con la edad. Crece en el sotobosque del norte de la provincia Paranense y del Chaco Santafesino, próximo a cursos de agua. Florece de enero a marzo, fructifica de marzo a junio. Observación: Las hojas y semillas se utilizan en medicina popular. Los frutos frecuentemente son parasitados por potreros modificados, banquinas y vías de ferrocarril. Florece desde septiembre a abril, fructifica desde diciembre a mayo. Observación: Sus hojas, frutos y semillas se utilizan en medicina popular (Barboza et al., 2006).

4. Senna occidentalis (L.) Link, Handbuch 2: 140. 1829.

Basónimo: Cassia occidentalis L., Sp. Pl. 1: 377. 1753.

Nombres vernáculos: "Café de Bonpland”, "Café cimarrón”, “Taperibá”, “Sen taperibá”.

Iconografía: Burkart (1946: 13: 38, Fig. 2); Jozami y Muñoz (1983: 143); Peña-Chocarro et al. (2006: 211).

Distribución geográfica: Dptos. General Obligado y La Capital.

Ejemplar de referencia: Brollo 361 (SF), 
Tedone 5099 (SF).

Hábito y ecología: Hierba anual, a veces subarbusto robusto y perenne, de 0,30 a 1,5 m de altura, de olor fétido. Habita en áreas del norte y centro de la provincia Paranense. Florece en primavera. Coleccionada con frutos en diciembre y marzo.

Observación: Especie frecuentemente cultivada como ornamental, medicinal y para la obtención de sus semillas que, tostadas y molidas, se utilizan como sucedáneo del café. Es considerada una especie tóxica para rumiantes, equinos, cerdos y aves de corral (Ragonese, E. \& V. Milano, 1984).

5. Senna pendula var. paludicola $\mathrm{H}$. S. Irwin \& Barneby, Mem. New York Bot. Gard. 35: 393-394. 1982.

Basónimo: Cassia pendula Humb. \& Bonpl. ex Willd., Enum. Pl. 1: 440. 1809. Nombres vernáculos: “Cañafístula”, "Yerba del burro”, “Cañuto de pito”, "Pito cañuto", "Sen”, "Sen del campo".

Iconografía: Jozami \& Muñoz (1983: 140 y 142); Burkart (1987: 517, Fig. 232); Arbo \& Tressens: (2002: 43, Fig. 1-87); Hurrell et al. (2004: 167); Peña-Chocarro et al. (2006: 213). Distribución geográfica: Dptos. Garay, General Obligado, La Capital, San Javier, San Jerónimo, San Justo y Vera. Ejemplar de referencia: Ragonese 3611 (SF), Pensiero 8120 (SF), Ragonese 3446 (SF), Pensiero 4959 (SF), Franceschi 620 (UNR), D’Angelo 1009 (SF), Pire \& Llovet 622 (SF). Hábito y ecología: Arbusto glabro, ramoso, de 1,5 a $3 \mathrm{~m}$ de altura, a veces trepador, con ramas fistulosas, de olor fétido. Frecuente en bosques en galería de la provincia Paranense, próximos a arroyos, lagunas y ríos, e integra bosques de Prosopis L. (algarrobos), Vachellia Wight \& Arn., (acacias) y Celtis L.(tala) de la Cuña Boscosa, en la provincia Chaqueña. Florece y fructifica de diciembre a abril.
6. Senna scabriuscula (Vogel) H. S. Irwin \& Barneby, Mem. New York Bot. 35: 435. 1982. Basónimo:CassiaoccidentalisL.var.scabriuscula Vogel, Gen. Cass. Syn.:21-22. 1837. Iconografía: Burkart (1987: 513, Fig. 230). Distribución geográfica: Dptos. Garay, La Capital y Vera.

Ejemplar de referencia: D’Angelo 996 (SF), Pensiero 6487 (SF), Marino 2301 (SF). Hábito y ecología: Subarbusto perenne, erecto o ascendente, ramificado, hasta de 1 m de altura, de olor fétido. Crece en la Cuña Boscosa, de la provincia Chaqueña y en áreas del Espinal, en banquinas y suelos arenosos, próximos a arroyos. Florece en primavera, fructifica en el verano y principios del otoño.

7. Senna spiniflora (Burkart) H. S. Irwin \& Barneby, Mem. New York Bot. Gard. 35: 569. 1982

Basónimo: Cassia spiniflora Burkart, Darwiniana 7: 235.1946.

Nombres vernáculos: "Palo Mataco", "Timoyuyo", "Matorral”, "Barba de tigre”, "Timo", "Retama”.

Iconografía: Bravo (1978: 362, Fig. 4). Distribución geográfica: Dpto. 9 de julio. Ejemplar de referencia: Ragonese 3103 (SF).

Hábito y ecología: Arbusto o subarbusto erecto, de 0,5 a $3 \mathrm{~m}$ de altura, muy ramificado y espinoso. Poco frecuente en la provincia. Hasta el presente ha sido coleccionado en el noroeste del sector santafesino de la provincia Chaqueña, en suelos secos y generalmente salinos. Florece desde octubre a marzo. Coleccionado con frutos maduros en el mes de febrero.

Observaciones: El ejemplar Ragonese 3103, citado bajo el nombre de Cassia aphylla Cav., determinado por Burkart, (23-06-1938), corresponde a esta especie, endémica de Argentina (Bravo, 1978).

Dado la prolongada y vistosa floración de 
este arbusto, se lo recomienda para formar cercos vivos (Bravo, 1978).

Senna spectabilis (DC.) H. S. Irwin \& Barneby, Mem. New York Bot. Gard. 35: 600. 1982.

Basónimo: Cassia spectabilis DC. , Cat. Pl. Horti. Monsp. 90. 1813.

Nombres vernáculos: "Carnaval”, “Cañafístula macho”, "Ramo carnaval”. Ejemplar de referencia: Burkart 212691 (SI), coleccionado por Ragonese en marzo 1941.

Hábito y ecología: Árbol caducifolio, a veces de porte arbustivo, de 5 a $8 \mathrm{~m}$ de altura.

Observaciones: Especie citada como cultivada en Santa Fe (Ragonese 1941, sin mencionar localidad ni departamento).

\section{CONCLUSIONES}

El análisis de la información obtenida en la presente contribución, permite afirmar que: - Los taxones: Senna aphylla (Cav.) H. S. Irwin \& Barneby y Senna spectabilis (DC.) H. S. Irwin \& Barneby, son excluidos de la flora de la provincia.

- En lo que respecta a su distribución, las especies identificadas habitan, fundamentalmente, las provincias Chaqueña, del Espinal y Paranense. Sólo Parkinsonia aculeata y Gleditsia triacanthos han sido halladas en la provincia Pampeana. Senna spiniflora y Libidibia paraguariensis constituyen especies típicas de la provincia Chaqueña, mientras que Peltophorum dubium y Senna occidentalis son características de la provincia Paranense.

- Los géneros mejor representados son: Senna (7 especies), Chamaecrista (3 especies) y Gleditsia (2 especies).

- En cuanto al status de las especies reconocidas, se hallaron: 18 especies nativas, y 1 naturalizada (Gleditsia triacanthos L.). En lo referente al hábito, se registraron: árboles (4), arbustos (6), árboles o arbustos (3), hierbas perennes o subarbustos (2) y hierbas (4).

\section{AGRADECIMIENTOS}

A los doctores J. Pensiero y F. Robbiati por la lectura crítica del manuscrito. A M. Fabbroni, R. Fortunato y L. Galetti, por su disponibilidad en el suministro de sugerencias y/o material. A los directores de los Herbarios consultados. A los árbitros.

\section{BIBLIOGRAFÍA}

1.- ARBO, M.M. \& S. G.TRESSENS (eds.) 2002. Flora del Iberá. EUDENE. Corrientes, $613 \mathrm{p}$.

2.- BARBOZA G., J.J. CANTERO, C.O. NUÑEZ Y L. Ariza Espinar (Edit.). 2006. Flora Medicinal de la Provincia de Córdoba (Argentina). Museo Botánico, 1252 p.

3.- BIANCO, C. A. \& J.J. CANTERO. 1992. Leguminosae, en Las plantas vasculares del suroeste de la Provincia de Córdoba. Iconografía. Universidad Nacional de Río Cuarto, Editorial de la Universidad. 229 p. 1 map.

4.- BIANCO, C. A., J. J. CANTERO. C. O. NUÑEZ Y L. PETRINA. 2001. Fabaceae, en Flora del Centro de la Argentina. Iconografía. Universidad Nacional de Río Cuarto, Editorial de la Universidad, 291 p.

5.- BRAVO, L. D. 1978. El género Cassia en la Argentina, I. Serie Aphyllae Darwiniana 21, (2): 342-399.

6.- BRAVO, L. D. 1981. El género Cassia en la Argentina. III. Serie Pachycarpae Darwiniana 23 (1): 257-277.

7.- BRIDSON, G. D. R. (Compiler). 2004. Botanico-Periodicum-Huntianum (BPH-2). Pittsburg: Hunt Institute for Botanical Documentation. 
8.- BRUMMIT, R. K. \& C. E. POWELL (eds.) 1992. Authors of plants names. Royal Bot. Gard., Kew. 732 p.

9.- BURKART, A. 1936. Las especies Argentinas y Uruguayas del género Caesalpinia, Revista Argent. Agron. 3: 67-112.

10.- BURKART, A. 1946. Dos leguminosas cuyas semillas son substitutos del café. Rev. Argent. Agron. 13: 38.

11.- BURKART, A. 1952. Las Leguminosas argentinas, silvestres y cultivadas. Ed. ACME. Buenos Aires, 569 p.

12.- BURKART, A. 1967. Leguminosae. En A. L. Cabrera. (eds.), Flora de la Provincia de Buenos Aires. Colecc. Ci. Inst. Nac. Tecnol. Agropecu. 4 (3): 394-647.

13.- BURKART, A. 1987. Leguminosae. En N. S. Troncoso y N. M. Bacigalupo (eds.), Flora Ilustrada de Entre Ríos. Colecc. Ci. Inst. Nac. Tecnol. Agropecu. 6 (3ª): 442-738.

14.- BURKART, A. Y A. CARTER. 1976. Notas en el género Cercidium (Caesalpinioideae) en Sud América. Darwiniana 20 (3-4): 305-311.

15.- CABRERA, A. L. 1994. Regiones Fitogeográficas Argentinas. Enciclopedia Argentina de Agricultura y Jardinería. (2da ed.) 2 (1). Edit. ACME. Buenos Aires. 85 p.

16.- CHRISTENHUSZ, M.J.M. \& J.W. BYNG. 2016. The number of known plants species in the world and its annual increase. Phytotaxa 261(3): 201-217.

17.- DEMAIO P., O. KARLIN Y M. MEDINA. 2002. Árboles nativos del centro de Argentina. L.O.L.A. Buenos Aires. 210 p.

18.- DIMITRI, M. J., J. LEONARDIS Y J. S. BILONI. 1997 a. El nuevo libro del árbol. Tomo I. El Ateneo. Buenos Aires. XIII + 120 p.

19.- DIMITRI, M. J., J. LEONARDIS Y J. S. BILONI. 1997 b. El nuevo libro del árbol. Tomo II. El Ateneo. Buenos Aires. XIII + 124 p.

20.- EYNARD C., A. CALVIÑO Y L.ASHWORTH. 2017. Cultivo de plantas nativas. Propagación y viverismo de especies de Argentina central. Editorial de la UNC. Córdoba, 446 p.
21.- FABBRONI, M. 2010. Fabaceae Lindl. Tribu Cassieae Bronn, en Fl. Valle de Lerma 10 (2): 43 p.

22.- FORTUNATO, R. 1986. Revisión del género Bauhinia (Cercideae, Cesalpinioidea, Fabaceae) para la Argentina. Darwiniana 27 (1-4): 527-557.

23.- FORTUNATO, R. 1997. Fabaceae, parte 2. Subfam. Caesalpinioideae. Tribu III. Cercideae, en A. T. Hunziker (ed.), Fl. Fan. Argent. 33: 3-9. ProFlora-CONICET.

24.- GAGNON, E., A. BRUNEAU, C.E. HUGHES, L.P. DE QUEIROZ Y G.P. LEWIS. 2016. A new generic system for the pantropical Caesalpinia group (Leguminosae). PhytoKeys 71: 1-160.

25.- JOZAMI, J, M. \& J. DE D. MUÑOZ. 1984. Árboles y arbustos de la Prov. de Entre Ríos. (3ra. ed.) IPNAYS (CONICET-UNL). Santa Fe, 421 p.

26.- JUÁREZ DE VARELA F. 2001. Fabaceae Lindl. Subfam. Caesalpiniodeae (R. Br.) A. DC., en Fl. Valle de Lerma 6 (10): 4 p.

27.- HAENE E. Y G. APARICIO. 2001. 100 Árboles argentinos. Edit. Albatros. Buenos Aires, $126 \mathrm{p}$.

28.- HAWKINS, J. A., L. WHITE OLASCOAGA, C. E. HUGHES, J. L. R. CONTRERAS JIMENEZ Y P. MERCADO RUARO. 1999. Investigation and documentation of hybridization between Parkinsonia and Cercidium praecox (Leguminosae: Cesalpinioideae). Pl. Syst. Evol. 216: 49-68.

29.- HURRELL, J. A., Y H. B. LAHITTE. 2002. Biota Rioplatense VII. Leguminosas nativas y exóticas. LOLA. Buenos Aires, 320 p.

30.- HURRELL J. A., D. BAZZANO Y G. DELUCCHI. 2004. Biota Rioplatense IX. Arbustos 2. Nativos y exóticos. LOLA.Buenos Aires, $288 \mathrm{p}$.

31.- IRWIN H. S. \& R. C. BARNEBY. 1982. The American Cassiinae. A sinoptical revisión of Leguminosae.Tribu Cassieae, subtri-

Revista FAVE - Ciencias Agrarias 17 (2) 2018 | 
A. M. Luchetti et al.

bu Cassinae in the New World. Mem. New York Bot. Gard. 35 (1-2): 1-918.

32.- LPWG. 2017. A new subfamily classification of the Leguminosae based on a taxonomically comprehensive phylogeny. Taxon, 66 (1): 44-77. February 2017.

33.- LUCHETTI, A. M. 2014. Las Leguminosas Mimosoideas de la provincia de Santa Fe. Natura Neotropicalis 45/1 y 2: 21-43.

34.- MUÑOZ, J., P. ROSS Y P. CRACCO. 1993. Flora indígena del Uruguay. Edit. Agropecuaria Hemisferio Sur, Montevideo, 284 p.

35.- PARODI, L. R. 1987. Enciclopedia Argentina de Agricultura y Jardinería (3ra. ed.) 1 (1) (Ed. actualizada por M. J. Dimitri). Ed. ACME, Buenos Aires, 651 p.

36.- PENSIERO, J.F. \& M. R. DE LA PEÑA. 1999-2000. Flora y Avifauna de la provincia de Santa Fe. Talleres Gráficos El Litoral Argentino, Santa Fe, 384 p.

37.- PENSIERO, J. F., H. F. GUTIÉRREZ, A. M. LUCHETTI, E. EXNER, V. KERN, E. BRNICH, L. OAKLEY, D. PRADO Y J. P. LEWIS. 2005. Flora vascular de la provincia de Santa Fe. Claves para el reconocimiento de las familias y géneros. Catálogo sistemático de las especies. Dpto. Publicaciones de la Universidad Nacional del Litoral, 401 p.

38.- PEÑA-CHOCARRO M., J. DE EGEA JUVINEL, M. VERA, H. MATURO Y S. KNAPP. 2006. Guía de Árboles y Arbustos del Chaco Húmedo. (Ed. J. De Egea y M. Peña-Chocarro). The Natural History Museum, Guyra Paraguay Fundación Moisés Bertoni y Fundación Habitat y Desarrollo. Asunción, Paraguay, 291 p.

39.- RAGONESE, A. 1941. La vegetación de la provincia de Santa Fe (R. A.). Darwiniana 5: 369-416.

40.- RAGONESE, A Y V. MILANO. 1984. Vegetales y substancias tóxicas de la flora argentina. Enciclopedia argentina de agricultura y jardinería. Segunda edición. Editorial
ACME S.A.C.I. 413 p.

41.- ROBBIATI F., A. M. ANTON Y R. FORTUNATO. 2014. A New Synonym in Senna, Series Aphyllae (Leguminosae, Caesalpinioideae). Syst. Bot., 39 (4): 1120 - 1126.

42.- RUIZ LEAL, A. 1972. Flora popular mendocina. Deserta 3, 299 p.

43.- ULIBARRI, E. A. 1979. Las especies argentinas del género Hoffmanseggia Cav. (Legum. Caesalp.) Darwiniana 22 (1-3): 135-158.

44.- ULIBARRI, E. A. 1984. Hoffmanseggia Cav. en M. N. Correa (ed.) Fl. Patagónica, Colecc. Ci. Inst. Nac. Tecnol. Agropecu. (4b): 220-223.

45.- ULIBARRI, E. A. 1994. Subfamilia 2. Caesalpinioideae, en R. Kiesling, Vazquez, Mazzini (ed.) Fl. San Juan 1: 257-263. Bs. As.

46.- ULIBARRI, E. A. 1996. Sinopsis de Caesalpinia y Hoffmannseggia (Leguminosae-Caesalpinioideae). Darwiniana 34 (1-4): 299-348.

47.- ULIBARRI, E. A. 1997. Fabaceae, parte 1. Subfam. I. Cesalpiniodeae, parte 1. Descripción y clave de tribus. Tribu 1. Cesalpinieae, en A. T. Hunziker (ed.), Fl. Fan. Argent. 32: 3-26. ProFlora-CONICET.

48.- ULIBARRI, E. A. 2008. Los géneros de Caesalpinioideae (Leguminosae) presentes en Sudamérica. Darwiniana 46 (1): 69-163.

49.- ZULOAGA, F. O. Y O. MORRONE (eds.) 1999. Fabaceae, en Catálogo de las Plantas Vasculares de la República Argentina II. Dicotiledoneae (F-Z). Monogr. Bot. Missouri Bot. Gard. 74: 623-744.

50.- ZULOAGA, F. O. Y O. MORRONE \& M. J. BELGRANO (eds.). 2008. Catálogo de las Plantas Vasculares del Cono Sur (Argentina, Sur de Brasil, Chile, Paraguay y Uruguay) Monogr. Syst. Bot. Missouri Bot. Gard. 107. St. Louis.

51.- ZULOAGA, F. O. Y O. MORRONE (eds.). X-2018. Catálogo de la Flora del Conosur. Edición on line www.darwin.edu.ar/ Proyectos/ Flora Argentina. http://www.mobot.org/mobot/research/apweb/ X-2018.Missouri Bot. Gard. 